\title{
Fibular osteocutaneous free flap reconstruction of the mandible after resection of ossifying fibroma in a seven-year old child
}

\author{
Bartosz Mańkowski ${ }^{1,2}$, Joanna Bilska-Stokłosa ${ }^{1}$, Krzysztof Osmola1, \\ Marian Nowaczyk ${ }^{1}$, Tadeusz Półchłopek ${ }^{1}$
}

Ossifying fibromas (OSF) belong to a group of benign fibro-osseous neoplasms encountered almost exclusively in the craniofacial skeleton. The clinical course is typically asymptomatic. However, discomfort in the mouth begins to develop once the neoplasm progressively extends, causing an overgrowth of bone resulting in pain and facial asymmetry. The development of craniofacial bone tumors in children is quite a rare occurrence. In international medical literature, the described cases typically involve young adolescent patients, with very few case reports of craniofacial neoplasms in children under the age of seven [1, 2]. In the current case report, we present a seven-year-old patient with a biopsy-proven ossifying fibroma. The patient underwent a resection of the neoplasm with reconstruction. One of the major issues encountered in reconstruction of the mandible using a vascularized fibular flap in children is the growth potential of the fibula in comparison to the mandible, resulting in a symmetrical facial image. The use of a fibular osteocutaneous free flap in reconstruction of mandibular deficits in children is a favourable solution. The use of this type of flap permits functional restoration and significantly improved facial cosmesis. The union of the mandible with the integrated fibula is still a topic of ongoing discussion and one must be prepared for possible further orthognathic procedures after completing the growth of the craniofacial skeleton.

NOWOTWORY J Oncol 2017; 67, 1: 54-58

Key words: ossifying fibroma, fibular flap, mandible, microvascular anastomosis, fibula

\section{Introduction}

Ossifying fibromas (OSF) belong to a group of benign fibro-osseous neoplasms that are encountered almost exclusively in the craniofacial skeleton. The first description of the tumor was proposed by Menzel in the year 1872. Montgomery further described this group of odontogenic neoplasms in extensive detail and coined them ossifying fibromas.

The pathogenesis of ossifying fibromas has not been completely elucidated and the etiology is still currently rather confounding. Nevertheless, there are several proposed hypotheses of the underlying etiology of this neoplasm. One proposed hypothesis states that these neoplasms originate from the periodontal ligament. This ligament is a layer of connective tissue containing stem cells that can develop into several cell lineages. Alternative hypotheses for the development of these perplexing neoplasms include periapical periodontal tissues, traumatic origins and other irritants. In 2005, the World Health Organization systemically grouped these neoplasms and distinguished three variants of ossifying fibromas that included ossifying fibroma, juvenile trabecular ossifying fibroma and juvenile ossifying psammomatoid fibroma $[3,4]$. It should be emphasized that the commonly applied term peripherial ossifying fibroma is a non-cancerous reactive change and is not a variant of extra-skeletal ossifying fibroma.

${ }^{1}$ Department of Head and Neck Surgery, Poznań University of Medical Sciences, Poland

${ }^{2}$ Department of Trauma, Burns and Plastic Surgery, Poznań University of Medical Sciences, Poland 
Ossifying fibromas can develop at any age; however, the peak incidence is between the second and the fourth decade of life. Additionally, this neoplasm has a significant predilection for females and usually presents later in the third or fourth decade of their life. Conversely, in males it occurs less frequently and generally tends to present in the first and second decade of life. In the pediatric population, this neoplasm had been previously localized in the fibula but it is much more commonly observed in the head and neck region. In approximately $62-89 \%$ of patients, this neoplasm presents in the mandible. Less common locations of development include the jaw, perinasal sinuses and the nasopharynx. Additionally, cases have been reported in the literature of localized changes in the bones of the cranial vault $[5,6]$.

The clinical course of this tumor is rather asymptomatic until the neoplasm increases in size and extends into the bone, causing discomfort in the mouth, pain and facial asymmetry. Bleeding into the oral cavity from the tumor may also occur. Rapid growth of the tumor may indicate an aggressive nature of the cancer. However, despite its rather benign course, an expanding neoplasm may cause significant destruction of skeletal tissues.

The diagnosis is generally made incidentally during dental examinations, routine radiologic examinations or craniofacial/head CT scans that were performed for unrelated reasons. The treatment of this neoplasm generally consists of a resection that is associated with extensive reconstructive surgical procedures. Following complete resections of the tumor, the prognosis is excellent. However, the neoplasm has tendencies to reoccur. Therefore, systemic clinical and radiologic examinations are indicated following surgical resections.

\section{Case report}

A seven-year-old girl was referred to our clinic because of rapidly growing mass on the right side of her mandible. On clinical examination the mass was hard and palpable in the buccal cavity. The mass was painless but it did cause significant discomfort to the child while eating. Subsequently, the mass was observed for approximately four months until initial treatment was started.

A CT scan was performed and an incisional biopsy of the tumor was completed (Fig. 1). The biopsy was examined and the diagnosis was confirmed histopathologically. Following histopathological confirmation, the tumor was removed under general anesthesia. The submandibular approach was utilized to unveil the tumor (Fig. 2A). However, because of the sheer size of the mass, we decided to do a segmental resection of the mandible. This included resection of the tumor with safe margins of healthy bone on both sides of the jaw (Fig. 2B). The oral mucosa was left intact and it separated the operative site from contact with the environment of the oral cavity. In total, approximately $6.5 \mathrm{~cm}$ of the mandible

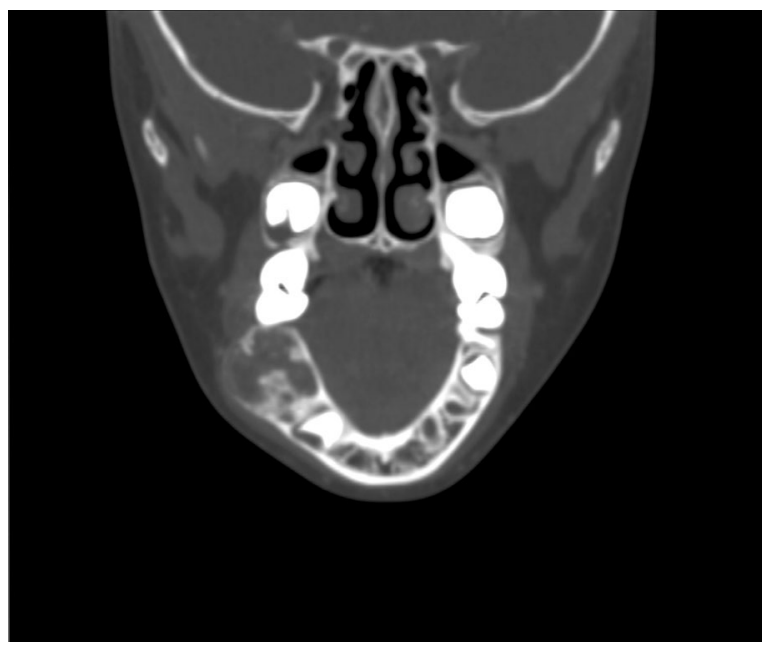

Figure 1. A CT scan of the mandible of the patient. Imaging shows a mass on the right side of the mandible

was resected. Simultaneously during the procedure, a fibular pedunculated free flap was harvested without a skin paddle. Next, we transferred the fibula into the resected tumor site and anastomosed the fibula to the mandible using a 10-hole mandible mini plate (Fig. 3). A microvascular anastomosis of the fibular artery to the facial artery using the end-to-end technique was performed. Additionally, the fibular vein was anastomosed to the internal jugular vein using the end-to-side technique.

The postoperative course of the patient progressed very well without any complications. The potency of the anastomosis was reviewed three times daily using an $8 \mathrm{~Hz}$ flowmeter vascular head. The child was admitted to the pediatric intensive care unit for three days, followed by an eight-day observation in maxillofacial surgery department. Six months after the reconstructive surgery and confirmation of bone union (Fig. 4), we removed the mandible mini-plate from the patient. The patient has been monitored in our outpatient clinic for a year with no evidence of recurrence. Following the surgery, she underwent physical rehabilitation for her lower extremity due to the fibular resection and is currently under the continuing care of an endodontist.

\section{Discussion}

Neoplasms in the mandible are fairly uncommon in children. Most of the tumors described in literature apply to children over eight years of age. There are only a limited number of reports that describe these neoplasms in children under seven years of age. Also, there is a greater occurrence of benign neoplasms than malignant ones [1,7]. Malignant tumors are reported to encompass up to $10 \%$ of all tumors [7]. However, some authors report an occurrence of 2.5, 2.9 and $7 \%[1,7-9]$. 


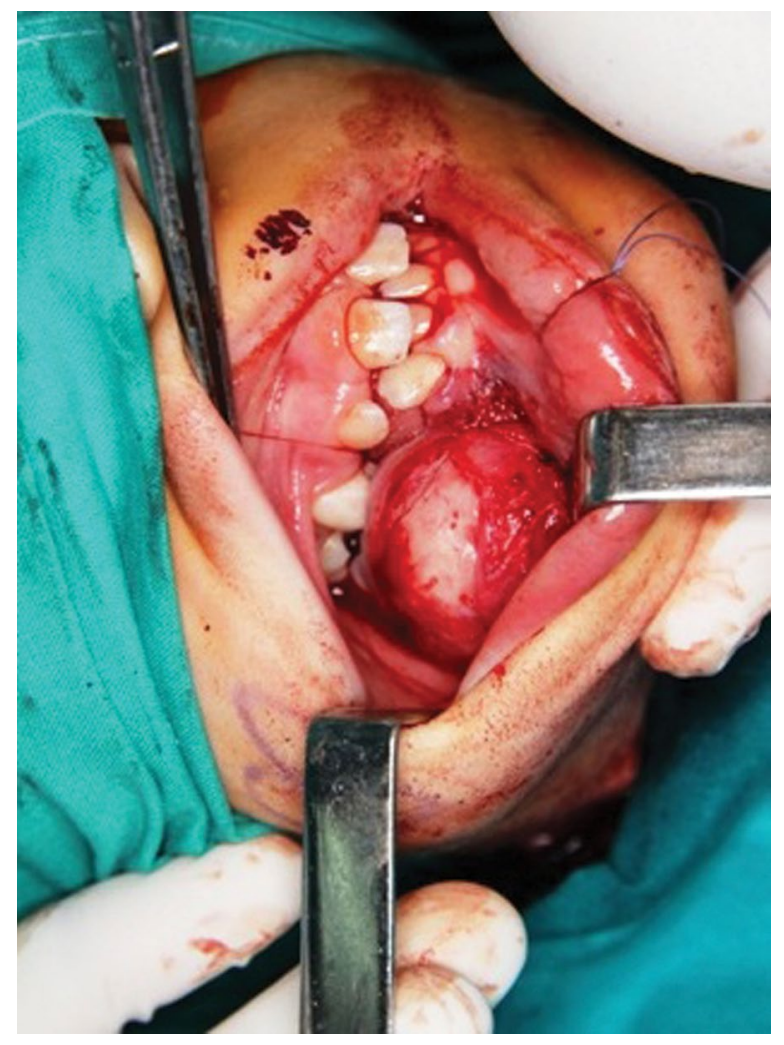

Figure 2A. Intra-operative exposure of the ossifying fibroma

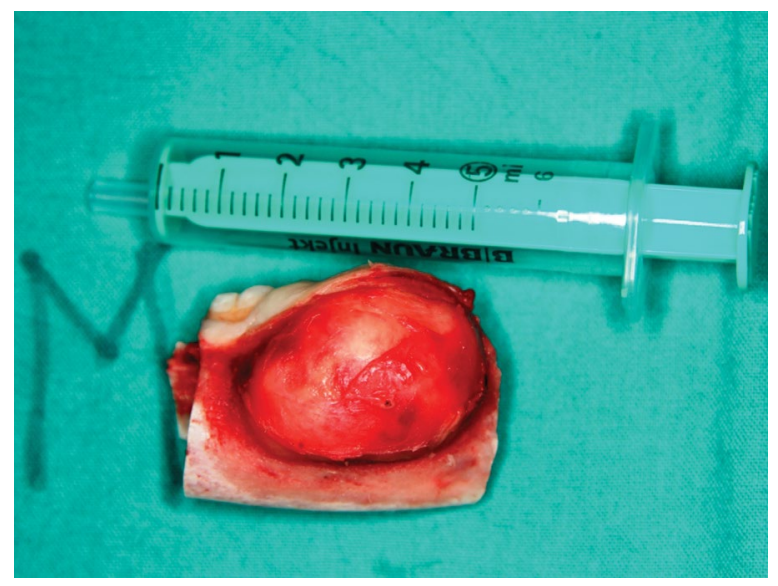

Figure 2B. The enucleated tumor following segmental resection of the mandible

Carcinogenic changes appear to develop in both bone and soft tissues. Tumors are most often asymptomatic and an unsettling soft tissue mass in the oral cavity is the most common reason for the child's complaint. Prior to surgical resection, intra-oral examinations and imaging studies are performed diagnostically. The tumor diagnosis should be confirmed by an incisional biopsy. Localization of the mass, its histology and visualization on imaging studies permit proper planning of surgical treatment.

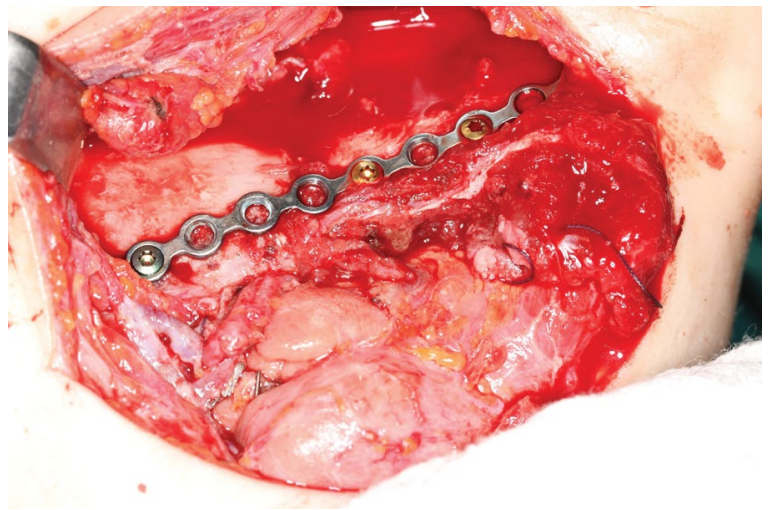

Figure 3. Intra-operative image of the reconstructed mandible with the exposed 10 hole mini-plate

Surgical management in cases of OSF usually consists of the enucleation of the tumor. However, if significant changes causing considerable skeletal destruction are evident, then a segmental resection of the mandible with simultaneous mandibular reconstruction is indicated. In pediatric cases, it appears that the best surgical option after resection of the mandible is a vascularized fibular flap. Microsurgical procedures in children are more difficult due to the smaller size of soft tissues, the smaller caliber of vessels for anastomoses, and a small operating field in the oral cavity. However, this approach using a free flap based microsurgical anastomosis carries fewer complications due to several factors. In children, the vessels are free of atherosclerosis and young patients are generally healthy. Additionally, children are not as commonly affected with diseases that have implications on the healing of soft tissues following surgery $[2,10,11]$. Guo et al. developed a surgical treatment algorithm for reconstruction in the case of mandible resection in children depending on their age. For children aged 7-8 years, they recommended reconstructive surgery using a free vascularized fibular flap, similar to recommendations in treatment of older children. Following resection in younger children aged 1-2 years it is recommended to use a temporary reconstructive plate followed by reconstruction from avascular bone. However, only in the event of failure was the use of a vascularised fibular flap recommended [2, 12]. Lastly, Warren et al. indicate use of a scapular/parascapular flap instead of a fibular flap as an option for reconstruction of mandibular defects in children. Similarly to in adults, following the use of a parascapular flap it is not possible to work with two teams consisting of a resection and reconstructive team dissecting the flap simultaneously [10]. Both authors describe the lack of complications after grafting of fibular flaps. The use of a fibular flap does not appear to cause growth defects or inefficiencies in gait and limb function [10-13]. 


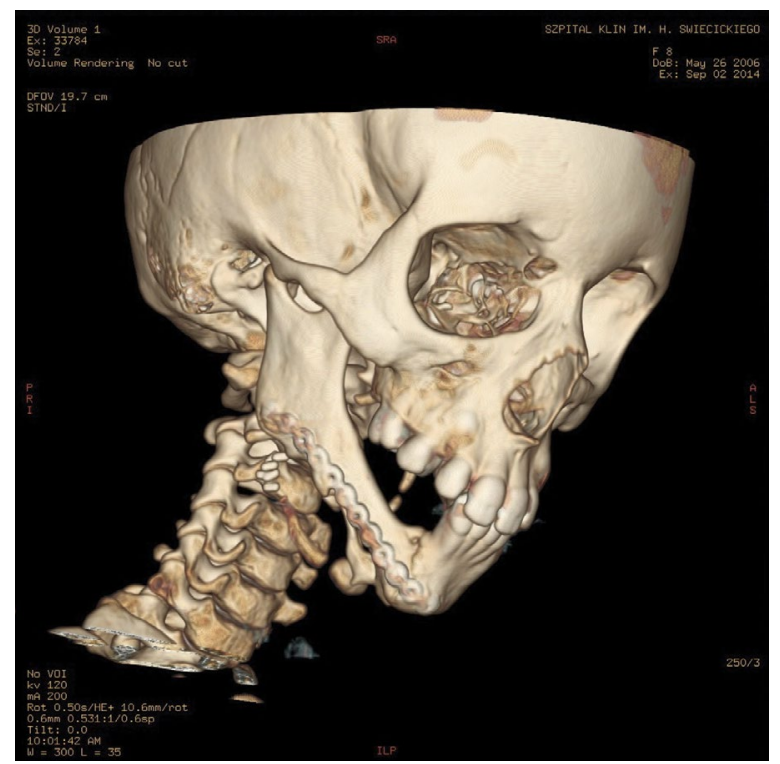

Figure 4. Three dimensional CT imaging with evident 10-hole mandible mini-plate with confirmation of bone union

One of the major problems encountered in reconstruction of the mandible using a vascularized fibular flap in children is the adequate growth potential of the fibula with the mandible to develop a symmetric facial image. Craniofacial development is dynamic and adequate growth potential is essential in permitting the development of proper facial symmetry. Experimental studies have shown that the transfer of fibular tissue with the growth plate does not impair its growth. However, some research has shown that the transfer of the central part of fibula without the metaphyseal growth plate to the mandible results in an inability of the fibula to grow. Therefore, an intimate understanding of growth center anatomy and its role in normal development is pivotal in allowing proper cranio- facial development and preventing functional deficits in the future.

Wietzman et al. described a very good functional and aesthetic effect after reconstruction of the mandible using a fibular flap along with proper and symmetrical growth of the mandible $[2,12,14]$. Additionally, Rashid et al. performed eighteen reconstructions of the mandible using fibular flaps in children with benign tumors of the mandible. The observations one to two years following surgical management showed no facial asymmetry and good light contour [15]. The problem of synostosis of transferred fibula to the mandible is still currently an ongoing area of discussion. Crosby et al. believe that the growth potential of the mandible is preserved when the remaining growth plate in the mandibular condyle area is left intact and the embedded fibular flap will adapt to the growth process which will not adversely affect the bite of the patient [16]. The authors also emphasize the difficulty in differentiating the elements of fibula in the mandible following synostosis on imaging studies [16]. Conversely, other authors such as Upton, Guo did not observe fibular growth after transfer to the mandible in cases of flaps that did not contain metaphysis. They observed facial skeleton asymmetries and in order to restore proper skeletal ratio, distraction osteogenesis or sagittal splitting of the mandible was recommended $[2,11]$. Additionally, Phillips et al. recommended that the annual period of observation of the child is not enough to draw conclusions about the growth of the fibula following transference to the mandible. Some researchers also recommend removing reconstruction plates following proven bone union to promote further growth and remodeling. In some children that underwent reconstructive surgery after resection of the mandible, the fibula reaches the age of puberty and bone growth ended. At this point it is important to assess disproportionate growth and consider orthodontic and orthognathic intervention.

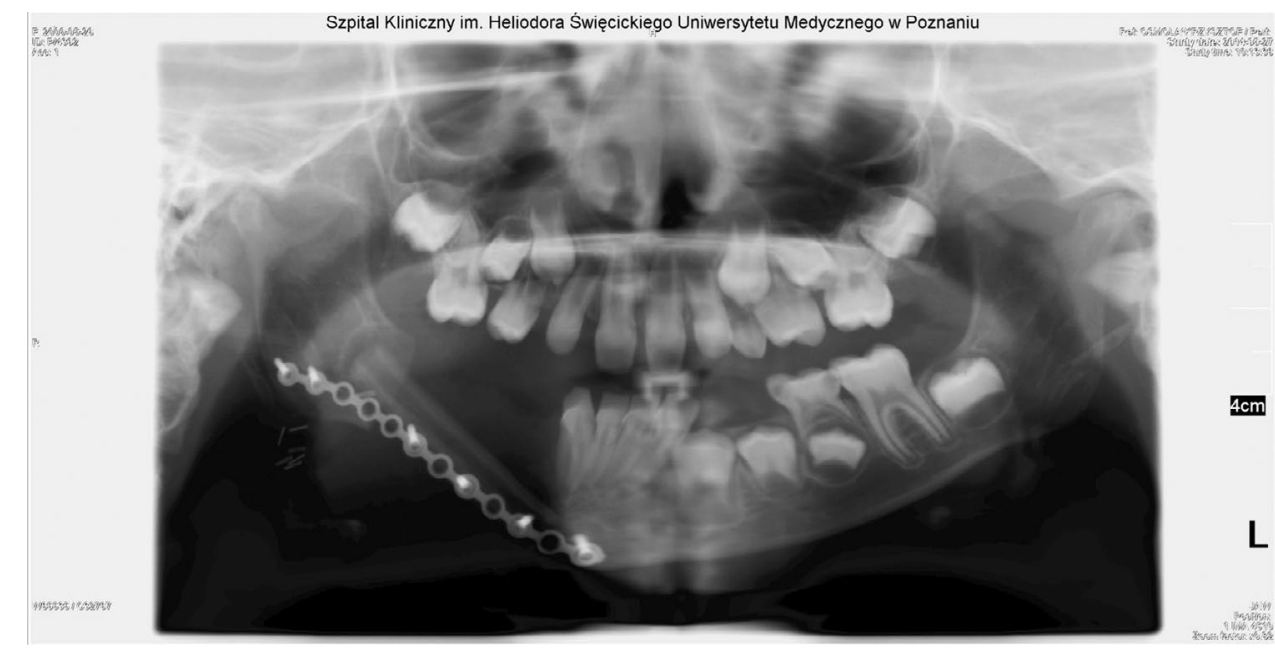

Figure 5. Panorex radiograph 14 days following surgery with visible 10-hole mandible mini-plate 
It appears that performing bilateral fibular osteotomy of the mandible with or without Le Fort 1 osteotomy of the jaw following the end of bone growth will provide the proper conditions of occlusion [12]. The authors emphasize not to reduce the angle of the mandible with osteotomy of the fibular graft during the procedure, because it may cause difficulty in potential corrective osteotomy in the future following completion of bone growth and may weaken the blood supply to the flap. As a result, this can potentially result in malunion of the fibular graft. In addition, it is recommended to resect a longer fibular flap and to impose it on the jaw with a longer fragment so that a sagittal fibular osteotomy can be performed in the future at this site. This will provide greater stability after osteotomy [12]. In addition to the reconstructive approaches that utilize bone flaps, Liu et al. proposed treatment of mandibular defects after removing the ameloblastoma using disc transportation distraction osteogenesis (TDDO) in children at the age of 23 months. This technique is also applicable for adults; however, it also proven to be effective in young patients. Distraction lasted 58 days and after 6 months the distraction device was removed. Proper reconstruction of bone and regeneration was observed. A slight shift of the mandible to the left approximately $2 \mathrm{~mm}$ during the distraction was discovered. However, no further mandibular asymmetry progression was seen. The regenerated bone served as a good substrate for the deposition of the dental implant [17].

\section{Conclusion}

In conclusion, our experience with the use of a fibular osteocutaneous free flap in the reconstruction of mandible deficits in children is a favourable solution. The flap permits functional restoration and a significant improvement of cosmetic facial appearance. However, microsurgical procedures in children require extensive experience with microscopes due to the small caliber of blood vessels. The union of the mandible with the integration of the fibula continues to be an ongoing topic of discussion and one must be prepared for possible further orthognathic procedures following complete growth of the craniofacial skeleton.
Conflicts of interests: none declared

\section{Tadeusz Półchłopek, MD}

Department of Trauma, Burns and Plastic Surgery

Poznań University of Medical Sciences

Fredry 10, 61-701 Poznań

e-mail:mdpolchlopek@gmail.com

Received: 31 Jan 2017

Accepted: 3 Mar 2017

\section{References}

1. Perry KS, Tkaczuk AT, Caccamese JF Jr. et al. Tumors of the pediatric maxillofacial skeleton: a 20-year clinical study. JAMA Otolaryngol Head Neck Surg 2015; 141: 40-44.

2. Guo L, Ferraro NF, Padwa BL et al. Vascularized fibular graft for mandibular reconstruction in pediatric patients. Plast Reconstr Surg 2008; 121: 2095-2105.

3. Barnes E, Evenson JW, Reichart P et al. (eds.). Pathology and genetics of head and neck tumors. Lyon: IARC Press; 2005: 319-321.

4. Hoang MP, Nguyen TT, Nguyen LK et al. Ossifying fibroma of the mandible: a case report using vascularized free fibula flap reconstruction. Plast Reconstr Surg Glob Open 2015; 3: e470.

5. Young N, Rowson JE. Cementifying fibroma of the frontal bone: a case report. Br J Oral Maxillofac Surg 2007; 45: 667-669.

6. Takaya M, Moritake $\mathrm{K}, \mathrm{Nagai} \mathrm{H}$ et al. Ossifying cementicular fibroma of the orbitofrontal bone in a child: case report. Noshuyo Byori 1993; 10: 87-90.

7. Aregbesola SB, Ugboko VI, Akinwande JA et al. Orofacial tumours in suburban Nigerian children and adolescents. Br J Oral Maxillofac Surg 2005; 43: 226-231.

8. Sato M, Tanaka N, Sato T et al. Oral and maxillofacial tumours in children: a review. Br J Oral Maxillofac Surg 1997; 35: 92-95.

9. Tanaka N, Murata A,Yamaguchi A et al. Clinical features and management of oral and maxillofacial tumors in children. Oral Surg Oral Med Oral Pathol Oral Radiol Endod 1999; 88: 11-15.

10. Warren SM, Borud LJ, Brecht LE et al. Microvascular reconstruction of the pediatric mandible. Plast Reconstr Surg 2007; 119: 649-661.

11. Upton J, Guo L, Labow BI. Pediatric free-tissue transfer. Plast Reconstr Surg 2009; 124 (6 Suppl): e313-326.

12. Phillips $\mathrm{JH}$, Rechner $\mathrm{B}$, Tompson BD. Mandibular growth following reconstruction using a free fibula graft in the pediatric facial skeleton. Plast Reconstr Surg 2005; 116: 419-424; discussion 425-426.

13. Upton J, Guo L, Labow BI. Pediatric free-tissue transfer. Plast Reconstr Surg 2009; 124 (6 Suppl): e313-326.

14. Weizman N, Gil Z, Wasserzug O et al. Surgical ablation and free flap reconstruction in children with malignant head and neck tumors. Skull Base 2011; 21: 165-170.

15. Rashid M, Tamimy MS, Ehtesham-Ul-Haq et al. Benign paediatric mandibular tumours: experience in reconstruction using vascularised fibula. J Plast Reconstr Aesthet Surg 2012; 65: e325-331.

16. Crosby MA, Martin JW, Robb GL et al. Pediatric mandibular reconstruction using a vascularized fibula flap. Head Neck 2008; 30: 311-319.

17. Liu Y, Chen J, Yan F et al. Mandible reconstruction with transport-disc distraction osteogenesis in children of deciduous dentition. Int J Oral Maxillofac Surg 2012; 41: 1223-1228. 